\title{
Bestimmung der Grundwasserneubildungshöhen für Festgesteinsgebiete in Niedersachsen
}

\author{
Gabriele Ertl ${ }^{1}\left[\right.$ D $\cdot$ Frank Herrmann ${ }^{2}$ (D) · Jörg Elbracht ${ }^{1}$ (ID \\ Eingegangen: 26. Juli 2021 / Überarbeitet: 22. November 2021 / Angenommen: 22. November 2021 / Online publiziert: 10. Januar 2022 \\ (c) Der/die Autor(en) 2021
}

\section{Zusammenfassung}

Die Grundwasserneubildung in Festgesteinsbereichen ist aufgrund der hydrogeologischen Eigenschaften der Gesteine im Allgemeinen geringer als in den Porengrundwasserleitern der Lockergesteine. Durch das Zusammenspiel dieser Eigenschaften mit der Topographie der Mittelgebirgslandschaften Niedersachsens, findet dort neben der Grundwasserneubildung auch ein signifikanter Zwischenabfluss statt. Methodisch können in Wasserhaushaltsmodellen im Rahmen einer Abflussseparation diese Größen mithilfe von flächendifferenzierten BFI-Werten berechnet werden. Dabei geben die BFI-Werte das Verhältnis von Grundwasserneubildung zum Gesamtabfluss wieder. Für die Abflussseparation mit dem Modell mGROWA wurden BFI-Werte für das Festgestein von Niedersachsen mit im Vergleich zu vorhergehenden Modellversionen höherem Detaillierungsgrad auf Basis der Geologischen Karte 1:50.000 (GK50) integriert. Dafür wurden 455 geologische Einheiten der GK50 hydrogeologisch analysiert und parametrisiert. Vor allem wasserwirtschaftlich relevante geologische Einheiten geringerer Ausdehnung konnten so besser in der Karte der Grundwasserneubildung berücksichtigt werden. Diesbezüglich werden fünf geologische Einheiten detaillierter diskutiert. Für diese fünf Fallbeispiele wurden mittels eines U-Tests die Zeitreihen der simulierten jährlichen Grundwasserneubildung auf signifikante Veränderungen geprüft. Es resultiert für alle Zeitreihen eine signifikant veränderte Verteilung der jährlichen Grundwasserneubildung auf einem geringeren Niveau in den gerade vergangenen beiden Dekaden.

Schlüsselwörter Grundwasserneubildung $\cdot$ Niedersachsen $\cdot$ Festgestein $\cdot$ mGROWA $\cdot$ Base Flow Index

\section{Determination of groundwater recharge for solid rock areas in Lower Saxony}

\begin{abstract}
Due to their hydrogeological characteristics, groundwater recharge rates in solid rock sites are typically lower than in the porous aquifers of unconsolidated rocks. The interaction of these characteristics with the topography of the low mountain range landscape of Lower Saxony leads to significant interflow in addition to groundwater recharge. In water balance models, these quantities can be calculated using runoff separation procedures by using BFI values. The BFI value specifies the ratio of groundwater recharge to total runoff. To separate runoff with the mGROWA model, we integrated BFI values for the solid rock sites of Lower Saxony with a higher level of detail than in previous model versions through the use of the Geological Map 1:50,000 (GK50). As a result, we obtained an improved representation of geological units with high relevance to water management but small extent in the spatial characterisation of groundwater recharge. For five case study units, we performed a U-test to assess changes and trends in the simulated time series of groundwater recharge. For all case study units we obtained a statistically significant reduced level of the annual groundwater recharge for the past two decades.
\end{abstract}

Keywords Groundwater recharge $\cdot$ Lower Saxony $\cdot$ Solid rock $\cdot$ mGROWA $\cdot$ Base Flow Index

Gabriele Ertl

gabriele.ertl@lbeg.niedersachsen.de

1 Landesamt für Bergbau, Energie und Geologie, Stilleweg 2, 30655 Hannover, Deutschland
2 Institut für Bio- und Geowissenschaften IBG 3 Agrosphäre, Forschungszentrum Jülich, Wilhelm-Johnen-Straße, 52428 Jülich, Deutschland 


\section{Einleitung}

Auch wenn Niedersachsen mit Niederschlagshöhen von im Mittel 787 mm/a (1981-2010, DWD 2018) größtenteils den humiden Klimaregionen zuzuordnen ist (Kottek et al. 2006), werden Daten zur Grundwasserneubildung in nahezu jedem wasserwirtschaftlichen Verfahren benötigt, um eine Übernutzung der Grundwasser-Ressourcen zu vermeiden. Die gegenwärtige Klimaveränderung mit steigenden Winterniederschlägen sowie steigenden Temperaturen (DWD 2018) führt darüber hinaus zu der Notwendigkeit, Grundwasserneubildung in höherer zeitlicher Auflösung und unter Beachtung der unterschiedlichen Standortfaktoren im Land genauer zu beleuchten (Herrmann et al. 2013; Scheihing 2019). Betrachtet man nur die Festgesteinsregionen Niedersachsens, die etwa ein Drittel der Landesfläche ausmachen, fällt im langjährigen Mittel der Niederschlag in einer räumlichen Spannbreite von 557-1817 mm/a (1981-2010, DWD 2018). Dabei muss bei der Bestimmung der Grundwasserneubildungshöhe eine breite Palette von Gesteinseigenschaften berücksichtigt werden.

Während die direkte Messung der Grundwasserneubildung generell nicht möglich ist, versucht man in Lockergesteinsgebieten punktuell zumindest die Sickerwasserhöhe mithilfe von Lysimetern zu messen. Dabei entspricht die Sickerwasserhöhe nur unter bestimmten Randbedingungen der Grundwasserneubildungshöhe. Je nach Standortbedingung teilt sich das Sickerwasser in Grundwasserzufluss und Zwischenabfluss auf. Lysimetermessungen geben auBerdem nur punkthafte Informationen, deren Qualität u.a. von technischen Randbedingungen der Anlage beeinträchtigt werden und die Übertragbarkeit der Daten ist nur bei exakt gleichen Standorteigenschaften wie z.B. identischer Bodenart oder Landnutzung gegeben (z.B. Beven 2000). Wittenberg et al. (2020) weisen auch auf teils erhebliche Unterschiede zwischen Punktniederschlag und Gebietsniederschlag in diesem Zusammenhang hin. Daher ist die Beurteilung von flächenhafter Grundwasserneubildung mithilfe von Lysimetermessungen schon im Lockergestein nur unter Beachtung aller Standorteigenschaften sinnvoll. Im Festgestein ist der Bau von Lysimetern nicht umsetzbar, da sich der Zustand von natürlich anstehendem Gestein nicht wiederherstellen lässt. Andere Berechnungen der Grundwasserneubildung z.B. mittels isotopenhydrologischer Verfahren existieren nur begrenzt für sehr kleine Einzugsgebiete (Herrmann und Schumann 2009), da hierfür umfangreiche Messreihen vorliegen müssen. Aus den genannten Gründen basieren die für wasserwirtschaftliche Zwecke erstellten Karten der Grundwasserneubildung heutzutage fast ausschließlich auf Simulationen mit Wasserhaushaltsmodellen.

Das Landesamt für Bergbau, Energie und Geologie (LBEG) stellt seit den 1980er-Jahren flächenhaft Daten zur Grundwasserneubildung für Niedersachsen bereit (z.B.
Dörhöfer und Josopait 1980). Seit 1998 wird für die flächendifferenzierte Ermittlung der langjährigen mittleren Grundwasserneubildung das Modell GROWA verwendet (Kunkel et al. 2006; Lemke und Elbracht 2008). Um die innerjährlichen Veränderungen der Grundwasserneubildung darstellen zu können, entwickelte das Forschungszentrum Jülich in Zusammenarbeit mit dem LBEG das Wasserhaushaltsmodell mGROWA (Herrmann et al. 2013). Durch methodische Verbesserungen am Modell sowie eine Aktualisierung mehrerer Datengrundlagen entstand die Modellversion mGROWA18 (Elbracht et al. 2018; Ertl et al. 2019; LBEG 2019).

In den GROWA-Modellen erfolgt im Rahmen einer rasterbasierten Abflussseparation die Abtrennung der Grundwasserneubildung vom Gesamtabfluss mithilfe des Konzeptes der „Base Flow Indizes“ (BFI). Auf diese Weise kann die räumliche Variabilität der Grundwasserneubildung im Festgestein unter Berücksichtigung der geologischen Eigenschaften modelliert werden (Bloomfield et al. 2009). Der BFI eines Einzugsgebietes stellt das langjährige Verhältnis von Basisabfluss zu Gesamtabfluss dar und liegt immer im Bereich zwischen 0 und 1. BFI können nur aus langjährigen gemessenen Abflussganglinien abgeleitet werden. Für die GROWA-Modelle müssen diese einzugsgebietsbezogenen BFI (Punktwerte an einem Pegel) in flächendifferenzierte BFI-Werte (Rasterwerte) regionalisiert werden, die den gesamten Festgesteinsbereich eines Modellgebietes lückenlos abdecken.

Dieser Beitrag behandelt die im Modell mGROWA verwendete räumliche Verteilung von BFI-Werten im Festgesteinsbereich Niedersachsens. Dazu wird auf die Methodik zur Bestimmung der BFI-Werte eingegangen und deren Entwicklungshistorie in Niedersachsen kurz dargestellt. Die verbesserte Darstellung der kleinräumigen Variabilität der BFI-Werte steht dabei im Mittelpunkt. Als räumliche Datenbasis diente die Geologische Karte 1:50.000 (GK50, LBEG 2007). Des Weiteren werden fünf geologische Einheiten als Fallbeispiele diskutiert, in denen die Bestimmung realistischer BFI-Werte besondere Bedeutung für lokale grundwasserwirtschaftliche Fragestellungen hat. Es ist jedoch nicht für alle geologischen Einheiten möglich, BFIWerte indirekt durch Basisabflusswerte zu validieren. Aus diesem Grund wird auch die Verwendung von Expertenwissen für eine Anpassung von als unsicher geltenden BFIWerten vorgeschlagen. Schlussendlich wird die räumliche Verteilung der mit mGROWA18 modellierten Grundwasserneubildung präsentiert und, aufgrund der Entwicklung in den vergangenen Jahren, die Variabilität und die Veränderungen in den Zeitreihen der Grundwasserneubildung in den exemplarischen geologischen Einheiten diskutiert. 


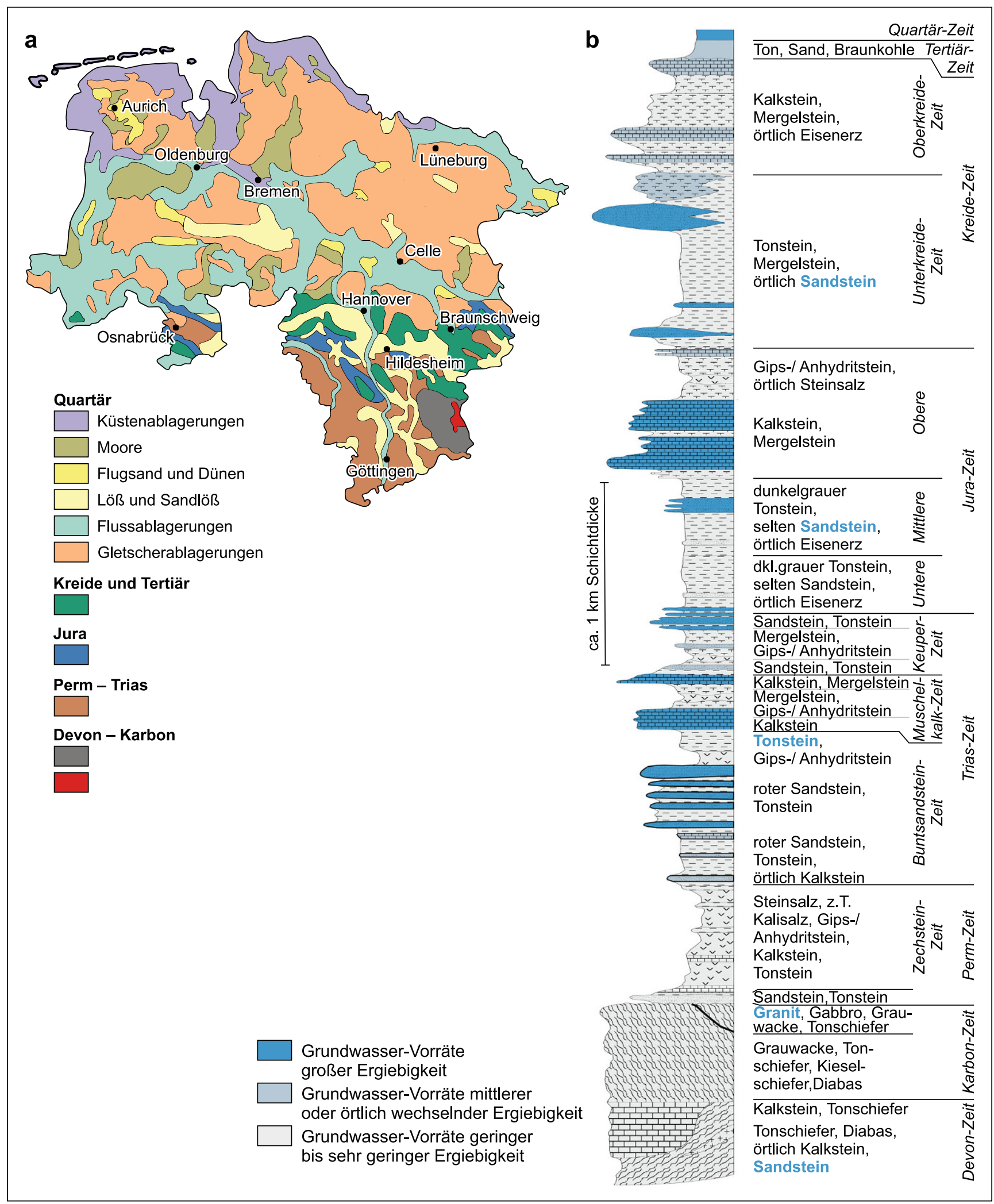

Abb. 1 a Geologische Übersichtskarte von Niedersachsen (Heunisch et al. 2007) und b schematisches hydrogeologisches Profil des Niedersächsischen Berglandes. Blau hervorgehoben sind die Gesteine der Fallbeispiele, an denen die Festlegung von BFI-Werten auf Basis der GK50 beschrieben wird (verändert nach Elbracht et al. 2016)

Fig. 1 a Geological overview map of Lower Saxony (Heunisch et al. 2007) and b hydrogeological profile of Lower Saxonys hillland. Highlighted (blue) are the units of the case studies where the determination of BFI based on GK50 is described (modified after Elbracht et al. 2016) 


\section{Hydrogeologie der niedersächsischen Festgesteinsgebiete}

Oberflächennah vorkommendes Festgestein gibt es in Niedersachsen vor allem im Niedersächsischen Bergland, das etwa ein Drittel der Landesfläche ausmacht (Abb. 1). Dabei werden die Festgesteinsschichten in diesen Bereichen weiträumig von meist sehr geringmächtigen jüngeren Lockergesteinsablagerungen überlagert. Nördlich der Linie Osnabrück - Hannover - Brauschweig tauchen die Festgesteine in größere Tiefen ab und kommen oberflächennah nur noch an wenigen Stellen z.B. über Salzstöcken vor (Heunisch et al. 2007).

Das Niedersächsische Bergland lässt sich in das präzechsteinzeitliche Grundgebirge und das überwiegend mesozoische Deckgebirge gliedern. Dabei treten Gesteine des Grundgebirges an der Oberfläche vor allem im Harz und in einigen kleinen Gebieten z. B. im Osnabrücker Bergland auf. Die geologischen Einheiten des Paläozoikums bestehen vor allem aus Tonstein, Kieselschiefer, Grauwacken und Sandstein sowie aus magmatischen Gesteinen (z.B. Brocken-Granit) (Ad-hoc-Arbeitsgruppe Hydrogeologie 2016).

Durch die variszische Orogenese gibt es in den paläozoischen Festgesteinen des Harzes einen ausgeprägten Großund Kleinfaltenbau. Hierdurch angelegte Kluftsysteme, Störungen, Schieferung und in Sedimentgesteinen auch die Schichtung bilden komplexe Wasserwegsamkeiten (Elbracht et al. 2016). Hinze (1971) beschreibt im Abstand von Zentimetern bis Dezimetern (selten Millimeter oder Meter) auftretende Ablösungsflächen. Eine tiefere Auflockerungszone liegt nach Hinze (1971) in den paläozoischen Gesteinen des Harzes im Allgemeinen 40-60m tief.

Die Lithologie des Gesteins hat einen weiteren Einfluss auf die hydraulische Leitfähigkeit in Kluftgrundwasserkörpern. Tonschiefer, Grauwacken und feinkörnige Sedimentgesteine verwittern zu lehmigen, relativ undurchlässigen Bodendecken. Die Klüfte dieser Gesteine sind meist durch zerriebenes Material aus Verwitterungsschutt verfüllt und abgedichtet. Im Allgemeinen gelten die Gesteine des Harzes daher als Grundwassergeringleiter. Hinze (1971) beschreibt für die Granite im Harz eine tiefgründige Vergrusung, die zur Bildung mehrere Meter mächtiger lockerer Kristallsande führt. Dadurch sind die Graniteinheiten auch als relativ gute Wasserleiter anzusprechen.

Das Deckgebirge des Niedersächsischen Berglandes bildet den Nordwest-Teil des Mitteldeutschen Bruchschollenlandes (Elbracht et al. 2016). Es besteht aus zechsteinzeitlichen und mesozoischen Gesteinen, die seit dem Jura zunehmend tektonischen Beanspruchungen ausgesetzt waren. Die Schichten wurden zu Antiklinalen und Synklinalen gefaltet und an zahlreichen Störungen zerbrochen. Dabei drang vor allem Steinsalz in die überlagernden Gesteine ein, woraus sich ein sehr uneinheitlicher und komplizierter Aufbau des Untergrundes und eine regional unterschiedliche tektonische Beanspruchung stratigraphisch gleicher Schichten ergibt.

Das Deckgebirge wird von einer bis über $6000 \mathrm{~m}$ mächtigen Folge von Festgesteinsschichten mit sehr unterschiedlichen hydrogeologischen Eigenschaften aufgebaut (Abb. 1). Durch den sehr heterogenen geologischen Aufbau sind auch die hydrogeologischen Verhältnisse wechselhaft und kompliziert. Grundwasser gibt es in unterschiedlicher Tiefe und in stark wechselnder Ergiebigkeit in Kluft- und teilweise auch in Karstgrundwasserleitern unterschiedlicher Ausdehnung. Im Vergleich zum Lockergestein sind die durchflusswirksamen Hohlraumanteile der Festgesteinsgrundwasserleiter, abgesehen von verkarsteten Bereichen, insgesamt eher gering. Grundwasservorkommen mit guter Ergiebigkeit gibt es in klüftigem Sandstein z.B. des Mittleren Buntsandstein oder der Bückeberg-Formation (Unterkreide) und in oft verkarstetem Kalk- und Mergelstein z. B. des Muschelkalks oder der Oberkreide. Aufgrund begrenzter Verbreitung bilden z. B. der „Cornbrash“-Sandstein des Mittleren Jura und die Kalksteinschichten des Oberen Jura lokal bedeutsame Grundwasserleiter (Elbracht et al. 2016).

Als Fallbeispiele für die Festlegung von BFI-Werten auf Basis der GK50 werden hier folgende Gesteine behandelt: Kahlebergsandstein-Formation (Devon), Brocken-Granit (Karbon), Gesteine des Röt (Trias), des „Cornbrash“ (Jura) und der Bückeberg-Formation (Kreide) (s. Abb. 1).

\section{Methodik}

\section{mGROWA Modellkonzept}

Mit mGROWA können die in Niedersachsen relevanten Komponenten des terrestrischen Wasserhaushaltes - die tatsächliche Evapotranspiration, der Gesamtabfluss mit seinen Direktabflusskomponenten (Zwischenabfluss, Drainageabfluss, urbaner Direktabfluss etc.) sowie die Grundwasserneubildung - in hoher räumlicher (100 m Raster) und zeitlicher Auflösung (Tage bzw. Monate) auf Landesebene über lange Zeiträume simuliert werden. Detaillierte Modellbeschreibungen sowie die dazu verwendeten Datengrundlagen sind in Herrmann et al. (2013) und Ertl et al. (2019) verfügbar und werden hier nur in den Grundzügen wiederholt. Die Simulation mit mGROWA ist in zwei Teile gegliedert. Im ersten physikalisch-basierten Teil erfolgt zunächst flächendifferenziert und instationär die Simulation der tatsächlichen Evapotranspiration und der gesamten Abflussbildung in Tagesschritten. Als Basis dient die allgemeine hydrologische Wasserhaushaltsgleichung. Der Speicherterm in dieser Gleichung wird durch das als Modul in mGROWA integrierte Mehrschicht-Bodenwasserhaushaltsmodell BOWAB 
(Engel et al. 2012) abgebildet. Die überschüssigen Wassermengen, die aufgrund der Wasserspeicherkapazität der Böden und versiegelter Oberflächen (z.B. in urbanen Räumen) nicht dauerhaft gespeichert werden können, werden als Gesamtabfluss bilanziert. Im zweiten empirisch geprägten Teil der Simulation wird dieser bilanzierte Gesamtabfluss im Rahmen einer rasterbasierten Abflussseparation in die oben genannten Komponenten aufgeteilt. Dabei kommen für die landesweit sehr heterogen verbreiteten spezifischen Standorteigenschaften jeweils angepasste Berechnungsmethoden zum Einsatz. Aufgrund der thematischen Bedeutung in diesem Artikel wird im Folgenden nur die Berechnung der Grundwasserneubildung anhand von flächendifferenzierten BFI-Werten im niedersächsischen Festgesteinsbereich genauer behandelt.

\section{Rasterbasierte Abflussseparation mit flächendifferenzierten BFI-Werten}

Die Grundwasserneubildung ist bei gleicher Höhe des versickernden Niederschlagsanteils in Festgesteinsbereichen meist geringer als in Lockergesteinsbereichen. Grund dafür sind die unterschiedlichen Charakteristiken der wasserdurchströmten Hohlräume. Im Festgesteinsbereich dominieren Kluft-Hohlräume, wobei jedoch in einigen Gesteinstypen auch relevante Poren-Hohlräume existieren, die meist jedoch nur einen geringen Anteil an der Wasserbewegung haben (z. B. einige Sandsteine im Buntsandstein). In stärker wasserlöslichen Gesteinen (z.B. Karbonatgesteinen) können außerdem Karst-Hohlräume ausgebildet sein. Zusätzlich existieren durch tektonische Beanspruchung entstandene Störungszonen mit kleinräumig stark variablen Kluftnetzen sowie durch Verwitterung, Mineralisationen oder Verschlämmung veränderte Kluftsysteme. Insgesamt führt die Genese und Evolution von Kluft- und Karstgrundwasserleitern $\mathrm{zu}$ einer teils sehr stark ausgeprägten Anisotropie der hydraulischen Leitfähigkeit, die jedoch auf der Maßstabsebene geologischer Karten räumlich nicht ausreichend genau abgebildet werden kann. In der Konsequenz führt diese Anisotropie (auch in Verbindung mit der Topographie an der Erdoberfläche der jeweiligen Formationen) zur Auftrennung der unterirdisch in den Deckschichten der Festgesteinsgrundwasserleiter strömenden Wassermengen in einen schneller abfließenden sogenannten Zwischenabfluss und in eine Grundwasserneubildung, die tiefere Bereiche erreicht, welche jedoch häufig geringe spezifische Speicherkoeffizienten aufweisen. Als Zwischenabfluss wird hier im Sinne von Bloomfield et al. (2009) sowie Haberlandt et al. (2001) die schnelle unterirdische Direktabflusskomponente verstanden, die nicht zur Grundwasserneubildung beiträgt.

Grundwasserneubildung und Zwischenabfluss sind in Festgesteinsbereichen nicht direkt messbar. Auch können physikalisch-basierte Modelle beide Größen in großen Modellgebieten aus Mangel an flächendeckender Information zur Anisotropie der hydraulischen Leitfähigkeit häufig nur näherungsweise ermitteln. Diese Information ist in diesen Modellen für eine Abtrennung lateraler Strömungskomponenten notwendig (Hintergrund dazu in Freeze and Cherry 1979). Aus diesem Grund werden in der Praxis weiterhin empirische Modellkomponenten genutzt, die sogenannte flächendifferenzierte BFI-Werte zur Charakterisierung der Auftrennung des unterirdischen Abflusses in Zwischenabfluss und Grundwasserneubildung verwenden.

Unter einem BFI versteht man das Verhältnis von Basisabfluss zum Gesamtabfluss. Über lange Zeiträume entspricht die Grundwasserneubildung unter Annahme eines stationären Speicherfüllstandes dem Basisabfluss. Aus diesem Grund kann über eine Abschätzung des langjährigen mittleren Basisabflusses eines Einzugsgebietes auf die Gebietsgrundwasserneubildung indirekt rückgeschlossen werden. Es resultieren in solchen Untersuchungen einzugsgebietsspezifische BFI. Wenn für Einzugsgebiete langjährige beobachtete Abflussganglinien verfügbar sind, kann mit einer Vielzahl mehr oder weniger komplexer Verfahren ein einzugsgebietsspezifischer BFI ermittelt werden, z.B. mit den Methoden beschrieben in Wundt (1958), Demuth (1993), Tallaksen (1995), Wittenberg (1999), Arnold und Allen (1999), Eckhardt (2008) oder Partington et al. (2012).

In Simulationen mit mGROWA können einzugsgebietsspezifische BFI noch nicht direkt verwendet werden. Es muss noch eine Übertragung auf die Rasterzellen anhand spezifischer Standorteigenschaften erfolgen. Diese Regionalisierung sollte Daten aus flächendeckenden Kartenwerken nutzen und erfolgt für Festgesteinsgebiete üblicherweise anhand der hydrogeologischen Gesteinscharakteristiken sowie unter Verwendung geeigneter Regionalisierungs-, Kalibrierungs- und Optimierungsalgorithmen (z.B. beschrieben in Kunkel und Wendland 1998; Haberlandt et al. 2001). Für die Festgesteinsgebiete in Niedersachsen wurde bereits in früheren Studien eine solche Regionalisierung vorgenommen. Dabei kam die im Folgenden erläuterte und ursprünglich für die Anwendung in Niedersachsen von Dörhöfer (2001a) vorgeschlagene Berechnungsmethode zum Einsatz. Als Ergebnis resultierten flächendifferenzierte BFI-Werte, wobei im Festgesteinsbereich die mittleren Basisabflüsse mit dem Verfahren von Demuth (1993) ermittelt wurden.

Die Ermittlung flächendifferenzierter BFI-Werte sollte möglichst auf einer großen Anzahl von Einzugsgebieten basieren, für die mit den oben angegebenen Methoden ein einzugsgebietsspezifischer $B F I_{\text {gem }}$ aus den Abflussganglinien verlässlich abgeleitet werden kann. Die Regionalisierung beginnt mit sinnvollen Annahmen für die flächendifferenzierten BFI-Werte der geologischen Einheiten $B F I_{H E}$, z.B. aufgrund der geohydraulischen Charakteristik. In ei- 
nem ersten Schritt werden aus diesen Annahmen und den Flächenanteilen $a$ der geologischen Einheiten in den jeweiligen Einzugsgebieten einzugsgebietsspezifische $B F I_{b e r}$ mithilfe der Gl. 1 berechnet. Im weiteren Verlauf der Berechnung werden diese $B F I_{b e r}$ den $B F I_{\text {gem }}$ gegenübergestellt und die $B F I_{H E}$ werden so lange modifiziert, bis entsprechend Gl. 2 die berechnete Summe der quadratischen Abweichungen zwischen den einzugsgebietsspezifischen $B F I_{b e r}$ und $B F I_{g e m}$ den kleinsten Wert (Min) annimmt.

$$
\begin{gathered}
\mathrm{BFI}_{\mathrm{gem}} \leftrightarrow \mathrm{BFI}_{\mathrm{ber}}=\sum_{i=1}^{n} \mathrm{BFI}_{H E, i} \cdot a_{i} \\
\sum_{j=1}^{m}\left(\mathrm{BFI}_{\mathrm{gem}, j}-\mathrm{BFI}_{\mathrm{ber}, j}\right)^{2}=M i n
\end{gathered}
$$

mit:

$B F I_{H E}$ flächendifferenzierter BFI-Wert einer geologischen Einheit $\{0 \ldots 1\}$

$B F I_{H E}$ einzugsgebietsspezifischer BFI aus der gemessenen Abflussganglinie abgeleitet $\{0 \ldots 1\}$

$B F I_{b e r} \quad$ einzugsgebietsspezifischer BFI aus Flächenanteilen berechnet $\{0 \ldots 1\}$

a Flächenanteile einer geologischen Einheit in einem Einzugsgebiet

$n \quad$ Anzahl der geologischen Einheiten

i Index der geologischen Einheiten

m Anzahl der Einzugsgebiete

j Index der Einzugsgebiete

Mit diesem Algorithmus werden jedoch meist nicht für alle geologischen Einheiten flächendifferenzierte BFI-Werte erzeugt, die sich in der Praxis als sinnvoll und richtig erweisen. Die Gründe dafür sind vielfältig. Beispielsweise resultieren Unsicherheiten aus den oben erwähnten Verfahren zur Bestimmung einzugsgebietsspezifischer BFI, die schwer zu quantifizieren sind und bei einer Übertragung auf kleine Gebietseinheiten in unbeobachteten Einzugsgebieten weitergegeben werden. So legt Eckhardt (2008) beispielsweise dar, in welcher Bandbreite BFI aus verschiedenen Berechnungsverfahren resultieren. Es hat sich deshalb in der Praxis insbesondere für flächenmäßig kleine geologische Einheiten mit einer lokal großen wasserwirtschaftlichen Relevanz als zielführend herausgestellt, durch Expertenwissen gestützte Korrekturen ausgewählter flächendifferenzierter BFI-Werte vorzunehmen. In großflächigen (landesweiten) Modellen stammen dementsprechend die räumlichen Verteilungen der BFI-Werte im Festgestein meist aus mehreren methodischen Ansätzen und Quellen. Letztendlich ist die Güte der flächendifferenzierten BFI-Werte entscheidend für die Qualität der Simulation der Grundwasserneubildung. Im Rahmen der rasterbasierten Abflussse- paration mit mGROWA erfolgt die Berechnung in einzelne Rasterzellen dann mithilfe der sehr einfachen folgenden Gleichung:

$q_{r}=B F I \cdot q_{t}$

mit:

$q_{r} \quad$ simulierte Grundwasserneubildung $[\mathrm{mm} / \mathrm{T}]$

$B F I \quad$ flächendifferenzierter BFI-Wert $\{0 \ldots 1\}$

$q_{t} \quad$ simulierter Gesamtabfluss [mm/T]

\section{Informationsbasis für die räumliche Verteilung der BFI-Werte}

Im Jahr 2006 ermittelten, kalibrierten und validierten Kunkel et al. (2006) mit der oben erläuterten Methodik BFIWerte zur Verwendung in der Modellversion GROWA06 für Einheiten der Hydrogeologischen Übersichtskarte von Deutschland 1:200.000 (HÜK200, BGR 2003). Die HÜK200 (BGR 2003) stellt den oberen großräumig zusammenhängenden Grundwasserleiter dar und basiert auf der Geologischen Übersichtskarte 1:200.000 von Deutschland. Dabei wurden geologische Gesteinseinheiten in $2 \mathrm{~m}$ Tiefe hydrogeologisch interpretiert und hinsichtlich ihrer hydrogeologischen Charakteristika attributiert (Dörhöfer et al. 2001b).

In den dann folgenden Modellversionen (vgl. Herrmann et al. 2013) wurden die im Festgestein verwendeten BFIWerte punktuell weiter angepasst und für die simulierte Grundwasserneubildung konnten gute ModellperformanceKennzahlen ermittelt werden. Die Genauigkeit der räumlichen Auflösung einer Übersichtskarte stellte sich für die Bestimmung der Grundwasserneubildung in mittelgroßen und großen hydrogeologischen Einheiten als angemessen heraus. Im Rahmen der Evaluierung der simulierten Grundwasserneubildung konnten jedoch auch schon einzelne Einheiten identifiziert werden, in denen größere Unsicherheiten bezüglich der Belastbarkeit der Ergebnisse bestanden, d.h. beispielsweise größere Differenzen zwischen der simulierten Grundwasserneubildung und den aus Abflussganglinien ermittelten Basisabflusswerten.

Mit Blick auf die wachsenden Anforderungen an die Modellversion mGROWA18, sollten die BFI-Werte für Niedersachsen in einen höheren Detaillierungsgrad überführt werden. Aus diesem Grund wurde für die Erstellung einer verbesserten räumlichen Verteilung der BFI-Werte im Festgesteinsbereich nun die Geologische Karte 1:50.000 (GK50, LBEG 2007) als Flächen- und Informationsbasis verwendet. Um den heterogenen strukturellen Bau des niedersächsischen Festgesteinsgebietes zu berücksichtigen, wurde darüber hinaus ein Fokus auf die strukturgeologische Bewer- 
Tab. 1 Übersicht der hauptsächlich auftretenden Festgesteine mit in mGROWA18 wirksamen BFI und Durchlässigkeitsbeiwerten (kf) nach Reutter (2011)

Table 1 Overview of the mainly occurring solid rocks in Lower Saxony including mGROWA18-BFI and hydraulic conductivity (kf) after Reutter (2011)

\begin{tabular}{|c|c|c|c|}
\hline Stratigraphie & Petrographie Hauptgemengteil & BFI & Kf nach Reutter (2011) [m/s] \\
\hline Devon-Karbon & $\begin{array}{l}\text { Diabas, Kieselschiefer, Tonschiefer, Gabbro, } \\
\text { Norit, Grauwacke }\end{array}$ & $0,05-0,25$ & $<1 \mathrm{E}-5$ \\
\hline Perm (Zechstein, Rotliegend) & Tonstein, Schluffstein, Rhyolit & & $<1 \mathrm{E}-5$ \\
\hline Trias (Oberer Buntsandstein, Keuper) & Tonstein, Schluffstein, Mergelstein & & $>1 \mathrm{E}-9-1 \mathrm{E}-5$ \\
\hline Jura & Tonstein, Schluffstein & & $>1 \mathrm{E}-9-1 \mathrm{E}-5$ \\
\hline Kreide & Tonstein, Schluffstein, Mergelstein & & $>1 \mathrm{E}-9-1 \mathrm{E}-5$ \\
\hline Tertiär & Tonstein (bis Ton) & & $<1 \mathrm{E}-5$ \\
\hline Devon-Karbon & Quarzit, Granit, Sandstein & $>0,25-0,50$ & $>1 \mathrm{E}-5-1 \mathrm{E}-4$ \\
\hline $\begin{array}{l}\text { Trias (Mittlerer u. Unterer Buntsandstein, } \\
\text { Mittlerer Muschelkalk, Keuper) }\end{array}$ & Sandstein, Schluffstein, Mergelstein & & $<1 \mathrm{E}-5-1 \mathrm{E} 4$ \\
\hline Jura & Sandstein, Mergelstein, Kalkmergelstein & & $>1 \mathrm{E}-5-1 \mathrm{E}-3$ \\
\hline Kreide & Sandstein, Mergelstein, Kalkmergelstein & & $>1 \mathrm{E}-7-1 \mathrm{E}-5$ \\
\hline Devon & Kalkstein & $>0,50-0,85$ & $>1 \mathrm{E}-5-1 \mathrm{E}-4$ \\
\hline Perm (Zechstein, Rotliegend) & Kalkstein, Konglomerat & & $>1 \mathrm{E}-5-1 \mathrm{E}-4$ \\
\hline $\begin{array}{l}\text { Trias (Mittlerer u. Unterer Buntsandstein, } \\
\text { Oberer und Unterer Muschelkalk, Keuper) }\end{array}$ & Sandstein, Kalkstein & & $>1 \mathrm{E}-5-1 \mathrm{E}-3$ \\
\hline Jura & Sandstein, Kalkstein, Quarzit & & $>1 \mathrm{E}-5-1 \mathrm{E}-3$ \\
\hline Kreide & Sandstein, Kalkstein & & $>1 \mathrm{E}-5-1 \mathrm{E}-4$ \\
\hline Tertiär & Sandstein & & $>1 \mathrm{E}-5-1 \mathrm{E}-3$ \\
\hline
\end{tabular}

tung und Einordnung der Gesteinseinheiten in ihren lokalen Ausprägungen gerichtet.

Die GK50 (LBEG 2007) vermittelt Informationen zur Verbreitung, Beschaffenheit und Abfolge von Gesteinen mit einer flächenhaften Verbreitung von mehr als $150 \mathrm{~m}^{2}$ bis in eine Tiefe von zwei Metern unter der Geländeoberfläche. Bei Festgesteinen lassen sich darüber hinaus in der Regel Rückschlüsse über die Fortsetzung dieser Gesteinsabfolgen in größere Tiefen ableiten. Basis der Kartenserie sind alle verfügbaren gedruckten Karten und Manuskriptkarten der Geologischen Karte von Niedersachsen 1:25.000 (GK25) sowie die daraus abgeleiteten digitalen Datensätze. Ferner wurden alle im LBEG verfügbaren geologischen Fremdkartierungen, alle vorhandenen Daten über Moore sowie von Fall zu Fall auch aktuelle Informationen aus den Bereichen Bodenkunde/Bodenschätzung in die Auswertung einbezogen.

Für die Verwendung in der Abflussseparation mit mGROWA18 wurden 455 Festgesteinseinheiten der GK50 flächendifferenzierte BFI-Werte zugewiesen. Dabei wurden auch Überlagerungsfälle bearbeitet, in denen quartärzeitliche Einheiten die kartierten Festgesteine abdecken, und es wurden die regionalen Ausprägungen der Gesteinsfazies sowie die strukturgeologischen Besonderheiten berücksichtigt. In einem ersten Schritt wurden die kalibrierten und validierten BFI-Werte der älteren GROWA/mGROWAModellversionen übernommen (Dörhöfer et al. 2001a; Wendland et al. 2003; Kunkel et al. 2006; Herrmann et al.
2013). Zusätzlich wurden aus Literaturquellen, Geländebeobachtungen und hydrogeologischen Gutachten Zusatzinformationen für eine nachträgliche Verbesserung der BFI-Werte in einzelnen geologischen Einheiten gewonnen. Dabei fiel auf, dass im Festgestein Niedersachsens auch kleinräumig auftretende Einheiten sowie lokale strukturgeologische Ausprägungen in der wasserwirtschaftlichen Praxis bedeutsam sind. In den kleinen Einzugsgebieten des Festgesteinsbereiches tragen teilweise nur eine oder wenige geologische Einheiten als ergiebige Grundwasserleiter zum Grundwasserdargebot bei. Treten diese Einheiten aufgrund steiler Lagerung oder geringer Schichtmächtigkeit nur mit geringer Ausstrichbreite auf, werden sie in großmaßstäbigen Karten nicht dargestellt. Ebenso bestimmen beispielsweise lokale Störungs- und Kluftsysteme maßgeblich die Wasserwegsamkeit und damit die Ergiebigkeit der Grundwasserleiter. Ist in der Fachliteratur oder in Detailkarten (z.B. Naumann 1927) lokal intensive Klüftung dokumentiert, wurde beispielsweise der BFI einer Einheit für diese Lokalität erhöht. Auf diese Weise konnten auch die nachfolgend als Fallbeispiele beschriebenen geologischen Einheiten mit der Verwendung der GK50 im landesweiten Modell praxisnäher und realistischer mit neuen BFIWerten parametrisiert werden. Sehr kleine BFI-Werte charakterisieren grundsätzlich beispielsweise Tonsteine und paläozoische Schiefergesteine. Verkarstung von lösungsanfälligem Gestein wie Kalkstein, Dolomit und Gips führt zu Karst-Grundwasserleitern. Grundsätzlich werden für 


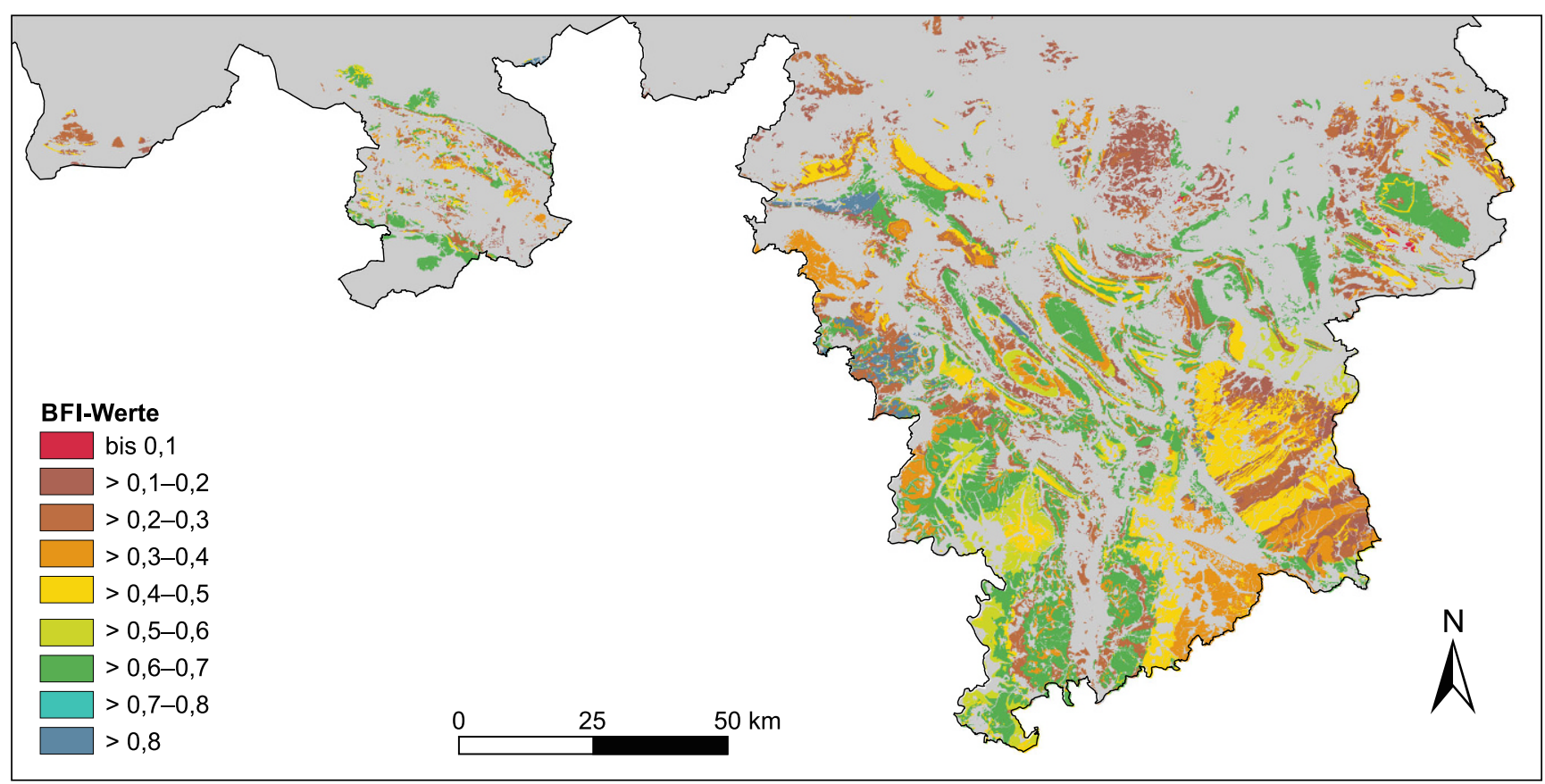

Abb. 2 Verteilung der BFI-Werte für Festgesteinseinheiten der GK50 in Niedersachsen

Fig. 2 Distribution of BFI values for GK50 bedrock units in Lower Saxony

Karstgebiete die höchsten BFI-Werte ermittelt, da intensive Verkarstung im Extremfall dazu führt, dass der gesamte Abfluss im Karst-Grundwasserleiter stattfindet. Mittlere BFI-Werte werden häufig für geologische Einheiten ermittelt, die von moderat tektonisch beeinflussten Sand-, Schluff-, und Mergelsteinen dominiert werden. Die Tab. 1 listet für die stratigraphischen Einheiten der GK50 und ihre Petrographie typische Bandbreiten der resultierenden BFIWerte auf. In Abb. 2 ist die räumlich präzisierte Verteilung der BFI-Werte im Festgesteinsbereich Niedersachsens dargestellt.

In Ertl et al. (2019) wurde die Wasserhaushaltssimulation mit mGROWA18 umfangreich evaluiert und es wurde in diesem Zusammenhang auch die Güte der landesweit berechneten Grundwasserneubildungshöhen bewertet und diskutiert. Herrmann et al. (2013) erläutern ebenfalls umfangreich die in Niedersachsen für mGROWA-Simulationen angewendete Evaluierungsmethodik und die damit verbundenen Unsicherheiten. Von den in Herrmann et al. (2013) dargestellten Einzugsgebieten liegen 22 mit Flächen von ca. $16-300 \mathrm{~km}^{2}$ in von Festgestein dominierten Gebieten. Insgesamt konnte in allen Studien eine gute landesweite Modellgüte ermittelt werden, wobei in einzelnen Einzugsgebieten immer auch größere Abweichungen zwischen simulierter Grundwasserneubildung und ermittelten Basisabflusswerten resultierten, deren Ursachen häufig nicht auf unrealistische BFI-Werte zurückgeführt werden konnten. Die Anpassung der BFI-Werte in den als Fallbeispiele beschriebenen geologischen Einheiten hat - aufgrund der La- ge der Einheiten und der Evaluierungs-Einzugsgebiete zueinander - keine relevante Auswirkung auf die gesamte Modellgüte. Für die Einzugsgebiete mit Vorkommen der Gesteinseinheiten der Fallbeispiele, für die im Nachhinein eine Bewertung anhand ermittelter Basisabflusswerte (nach Demuth 1993) möglich wurde, ist diese nachfolgend angegeben.

\section{Fallbeispiele}

\section{Kahlebergsandstein-Formation (Devon)}

Aufgrund der Gesteins- und Verwitterungseigenschaften gelten die Gesteine des Harzes ganz überwiegend als Grundwassergeringleiter, da sie häufig keine nennenswerte Matrixdurchlässigkeit haben und in Oberflächennähe Klüfte durch Verwitterungsbildungen abgedichtet sind. In Quarziten, Graniten und Diabasen wird keine oder wenig Verfüllung von Klüften durch abgeriebenes Material wie Verwitterungsprodukte beobachtet (Hinze 1971). Um dies zu berücksichtigen erfolgte eine Anpassung des BFIWertes auf 0,45 für die Quarzite des KahlebergsandsteinKomplexes.

\section{Brocken-Granit (Karbon)}

Aufgrund seiner Gesteinseigenschaften wird Granit üblicherweise als Grundwassergeringleiter eingestuft. Für die 


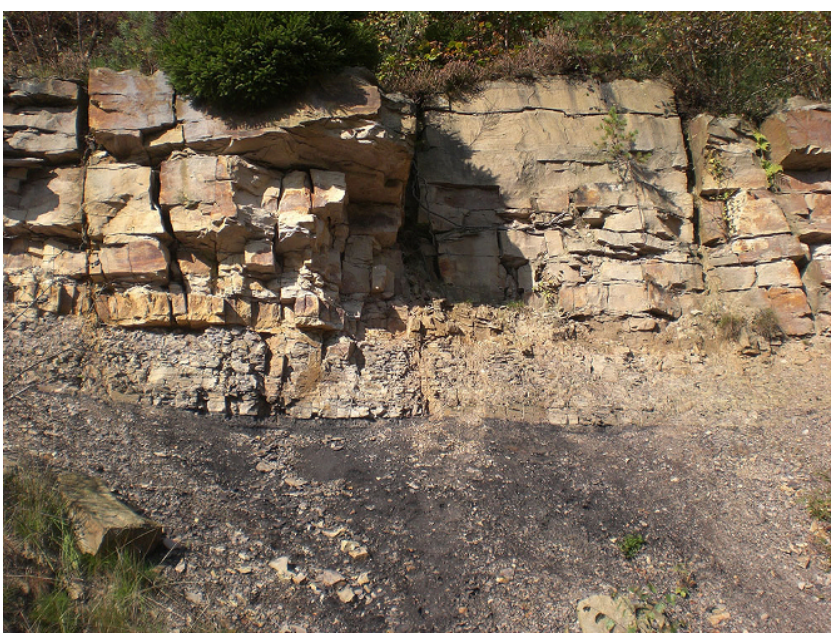

Abb. 3 Überlagerung von kohleführenden, gering durchlässigen Schichten durch geklüfteten Sandstein der Bückeberg-Formation an einem Weganschnitt am Wallmannsweg im Deister (Höhe des Bildausschnitts ca. $6 \mathrm{~m}$ )

Fig. 3 Coal-bearing, low permeability strata underlying fissured sandstone of the Bückeberg Formation at a road cutting at Wallmannsweg in the Deister (height of the picture approx. $6 \mathrm{~m}$ )

Granite im Harz beschreibt Hinze (1971) allerdings eine tiefgründige Vergrusung, die zur Bildung mehrerer Meter mächtiger lockerer Kristallsande führt, die gute Grundwasserleiter bilden. Um diese regionale Besonderheit zu berücksichtigen, wurden die BFI-Werte für die Graniteinheiten entsprechend angepasst, was für den Brocken-Granit zu einem im Vergleich zur Modellversion GROWA06 deutlich höher angesetzten BFI-Wert von 0,4 führt. Teile der Kahlebergsandstein-Formation (Devon) und des Brocken-Granit (Karbon) befinden sich im Einzugsgebiet des Pegels Schladen, für das ein Vergleich von langjähriger mittlerer Grundwasserneubildung (1971-2000) mit dem aus der Abflussganglinie abgeleiteten Basisabfluss möglich ist (ca. $170 \mathrm{~mm} / \mathrm{a}$ vs. ca. $200 \mathrm{~mm} / \mathrm{a}$ ). Die Erhöhung der BFIWerte hat hier zu einer besseren Anpassung der Grundwasserneubildung geführt. Generell sind jedoch solche Vergleiche in vielen Einzugsgebieten des Harzes mit größeren Unsicherheiten verbunden, weil insbesondere durch die zahlreichen Talsperren keine völlig unbeeinflussten Niedrigwasserganglinien vorliegen.

\section{Röt (Trias)}

Aufgrund der Generalisierung, die für die Darstellung in der HÜK200 (BGR 2003) erforderlich war, wurden Abfolgen des Oberen Buntsandstein (Röt) und des Muschelkalks häufig nicht differenziert dargestellt. Dabei wurde der sehr geringleitend eingestufte Ton- und Schluffstein des Oberen Buntsandstein teilweise mit den Grundwasserleitern des Unteren Muschelkalk zusammengefasst, die in der wasserwirtschaftlichen Praxis bedeutsam sind, sodass zum Bei- spiel entlang der Ränder des Leinetals keine realitätsnahen Grundwasserneubildungswerte ermittelt werden konnten. Durch die höhere räumliche Auflösung der GK50, die z. B. auf Kartierungen von Jordan et al. (1984) beruht, können die hydrogeologisch unterschiedlichen Einheiten nun differenziert dargestellt werden. Der BFI-Wert für Röt (Trias) beträgt 0,25 . Aufgrund der Verbreitung dieser Einheit ist eine explizite Bewertung der veränderten BFI-Werte anhand gemessener Abflussganglinien nicht möglich.

\section{„Cornbrash" (Jura)}

Die Gesteine des Mittleren Jura bestehen ganz überwiegend aus Ton- und Schluffstein, in die regional Kalksandsteinund Kalksteinschichten des „Cornbrash“ eingeschaltet sind. Durch ihre geringe Mächtigkeit bis etwa $30 \mathrm{~m}$ erreichen die „Cornbrash"-Schichten nur eine sehr geringe Ausstrichbreite, sodass sie in der HÜK200 (BGR 2003) nicht dargestellt werden konnten. Aufgrund ihrer wasserwirtschaftlichen Bedeutung in Gebieten, die ansonsten von den Grundwassergeringleitern des Mittleren Jura geprägt werden, hat dies zu Problemen in wasserrechtlichen Verfahren geführt. In der Grundwasserströmungsmodellierung mit Grundwasserneubildungswerten basierend auf nur einem mittleren BFI-Wert für Mittleren Jura konnte kein zufriedenstellendes Ergebnis erzielt werden (CONSULAQUA Hildesheim, Geo-Infometric 2015). Durch die Attributierung mit einem BFI von 0,6-0,7 im Gegensatz zu einem BFI von 0,15 für die in direktem Kontakt anstehenden Ton- und Schluffsteine konnte die Grundwasserneubildung für den Ausstrichbereich deutlich realitätsnäher modelliert werden. Aufgrund der Größe dieser Einheit ist eine explizite Bewertung der veränderten BFI-Werte anhand gemessener Abflussganglinien nicht möglich.

\section{Bückeberg-Formation (Kreide)}

Der Sandstein der Bückeberg-Formation bildet zahlreiche Höhenzüge im Weserbergland südlich von Hannover. Er ist häufig eng verzahnt mit Tonstein- und Kohleschichten (Abb. 3), sodass er zusammen mit den umgebenden Schichten bei der Attributierung auf der Basis der HÜK200 (BGR 2003) mit einem BFI von 0,2 versehen wurde. Da die Sandsteinschichten häufig geklüftet und wasserführend sind (Naumann 1927), hat diese Einstufung zu einer signifikanten Unterschätzung der Grundwasserneubildung und damit zu Problemen bei wasserwirtschaftlichen Fragestellungen geführt. In der Modellversion mGROWA18 beträgt der BFI-Wert nun 0,4. Ein Vergleich der nun für den Pegel Wunstorf resultierenden langjährigen mittleren Grundwasserneubildung (1971-2000) von ca. $83 \mathrm{~mm} / \mathrm{a}$ mit dem aus der Abflussganglinie abgeleiteten Basisabfluss von ca. $84 \mathrm{~mm} / \mathrm{a}$ unterstützt die neue Einstufung. Für den Pegel 


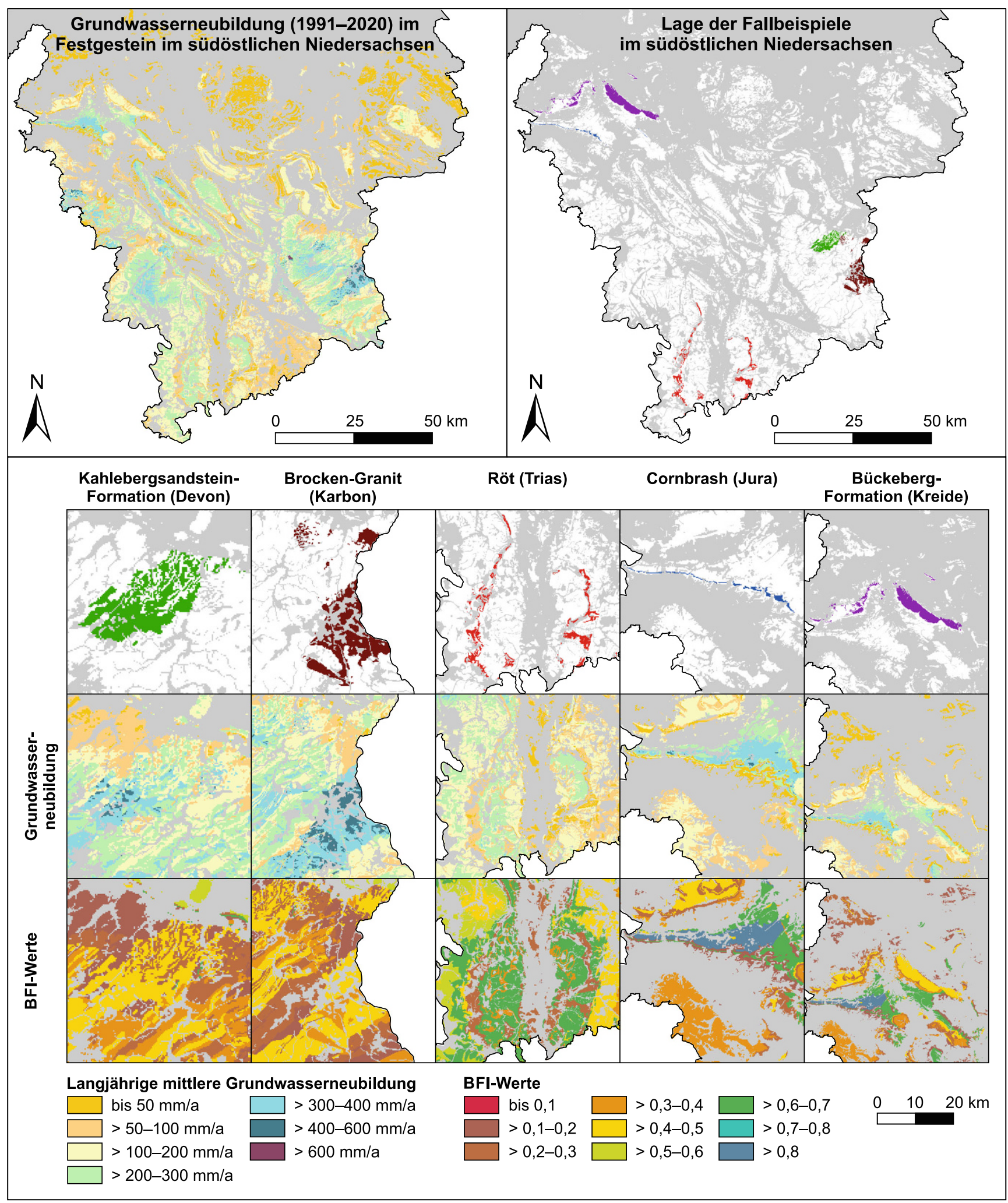

Abb. 4 Räumliche Verteilung der mit mGROWA bestimmten langjährigen mittleren Grundwasserneubildung (1991-2020) im Festgesteinsbereich im Südosten Niedersachsens. Zusätzlich gezeigt ist die Lage der als Fallbespiele diskutierten geologischen Einheiten sowie die räumliche Verteilung der BFI-Werte in ihrem Umfeld

Fig. 4 Spatial distribution of long-term mean annual groundwater recharge (1991-2020) in the solid rock area of Lower Saxony determined using mGROWA. Additionally shown are the locations of the example geological units and the spatial distribution of BFI values in their vicinity 
Düendorf wurde eine ähnlich gute Übereinstimmung gefunden (Grundwasserneubildung ca. $57 \mathrm{~mm} / \mathrm{a}$ vs. Basisabfluss ca. $60 \mathrm{~mm} / \mathrm{a})$.

\section{Räumliche Variabilität der Grundwasserneubildung}

Am LBEG liegen flächendeckend für ganz Niedersachsen Ergebnisse aus mGROWA18-Simulationen für die Zeit von 1961 bis 2020 vor. In Abb. 4 ist für die Festgesteinsbereiche im südöstlichen Niedersachsen die auf Basis der Simulationen berechnete langjährige mittlere Grundwasserneubildung der Periode 1991-2020 dargestellt. Zusätzlich ist auch die Grundwasserneubildung im Umfeld der Fallbeispiele vergrößert zu sehen. Deutlich sichtbar sind die aus der BFIWerte-Verteilung resultierenden kleinregionalen Kontraste, die zu einer Spannbreite der Grundwasserneubildung von 50 bis mehr als $400 \mathrm{~mm} / \mathrm{a}$ führen. In den Hochlagen der Mittelgebirge, insbesondere in denen des Harzes, werden die hohen Grundwasserneubildungshöhen auch durch die hohen Jahresniederschläge verursacht.

Für die Kahlebergsandstein-Formation (Devon) im nordwestlichen Harz resultieren ca. 300 mm/a. Für den BrockenGranit resultieren mit der Modellversion mGROWA18 je nach topographischer Lage und Exposition der Verbreitungsflächen mittlere Grundwasserneubildungshöhen zwischen 200 und $400 \mathrm{~mm} / \mathrm{a}$. Die geringsten mittleren Grundwasserneubildungshöhen unter den Fallbeispielen weist Röt (Trias) im Leinetalgraben mit lediglich etwa 30 bis $80 \mathrm{~mm} / \mathrm{a}$ auf. Für „Cornbrash“ (Jura) im Wesergebirge resultieren ca. 200 bis $260 \mathrm{~mm} / \mathrm{a}$ im Jahr sowie für die nördlich davon im Deister verbreitete Bückeberg-Formation (Kreide) nur ca. $120 \mathrm{~mm} / \mathrm{a}$.

\section{Zeitliche Variabilität der Grundwasserneubildung}

Hinter den in Abb. 4 dargestellten räumlichen Mustern der Grundwasserneubildung stehen mit mGROWA18 für jede 100-m-Rasterzelle zeitlich hochaufgelöst simulierte Zeitreihen. Für die Verbreitungsflächen der Gesteinseinheiten der Fallbeispiele sind diese Zeitreihen in Abb. 5 dargestellt. Der obere Teil der Darstellungen zeigt die Summe in den hydrologischen Jahren (November bis Oktober). Zusätzlich ist in Rot die Tiefpass-gefilterte Zeitreihe (Gauß-Filter, 10 Jahre) dargestellt, d.h. es werden dadurch die mittelfristigen Schwankungen hervorgehoben und längerfristige Trends deutlicher sichtbar. In Grau gestrichelt sind außerdem die langjährigen Mittelwerte der 20-Jahres-Perioden 1961-1980, 1981-2000 sowie 2001-2020 dargestellt. In der zweiten (unteren) Komponente der Darstellungen sind für die einzelnen hydrologischen Jahre die Monatssummen farblich kodiert dargestellt (Monate ohne Grundwasserneubildung in grau). Durch diese Darstellungsweise werden saisonale Muster und deren Veränderungen visualisiert.

Hinsichtlich der Niveaus und Amplituden der Grundwasserneubildung unterscheiden sich die Fallbeispiele teilweise sehr deutlich. Die zeitlichen Muster, d.h. die interannuelle Variabilität, periodische Phasen mit einem andauernden Bilanzdefizit der Grundwasserneubildung unterhalb des langjährigen Mittelwertes und langjährige Trends, sind jedoch weitgehend synchron ausgeprägt. In den geologischen Einheiten im Harz findet aufgrund der stark orographisch geprägten Niederschläge auch häufiger während der Sommermonate Grundwasserneubildung statt. In den topographisch tiefer liegenden Einheiten ist hingegen eine stärkere Saisonalität der Grundwasserneubildung sichtbar, d.h. der Hauptteil der Grundwasserneubildung findet im Winterhalbjahr statt. Bemerkenswert ist für alle Fallbeispiele ein etwa im Jahr 2000 einsetzender sukzessiver Rückgang, sowie ein Niveau, welches in der vergangenen Dekade unter dem Mittelwert der Periode 1981-2000 verbleibt.

Aufgrund dieser visuellen Interpretation der Zeitreihen der Grundwasserneubildung in Abb. 5 stellt sich die Frage, ob sich in den vergangenen Dekaden das Grundwasserneubildungsregime bereits verändert hat. Sie kann mithilfe eines statistischen Tests beantwortet werden. Als Test auf Signifikanz der Veränderung des Grundwasserneubildungsniveaus zwischen zwei Perioden wurde ein verteilungsfreier U-Test (Mann-Whitney-Wilcoxon-Test; Mann und Whitney 1947; Wilcoxon 1945) verwendet. Die Nutzung eines verteilungsfreien Tests hat den Vorteil, dass keine Normalverteilung der zu untersuchenden Größe notwendig ist. Bei stark klimatologisch beeinflussten hydrologischen Größen wie der Grundwasserneubildung ist eine Normalverteilung häufig nicht gegeben. Für die Analyse multidekadischer Regime-Verschiebungen von Abflusskomponenten wird der U-Test mittlerweile häufig angewendet (z. B. Mauget 2003).

Für die Fallbeispiele wurden mit dem U-Test die Perioden 1981-2000 und 2001-2020 auf Unterschiede geprüft. Es resultiert für alle Zeitreihen eine signifikant unterschiedliche Verteilung (Signifikanzniveau $\alpha=0,1$ ) der jährlichen Grundwasserneubildung auf einem geringeren Niveau in den gerade vergangenen beiden Dekaden. Die Anwendung des U-Tests auf die Perioden 1961-1980 und 1981-2000 ergibt, außer für die geologischen Einheiten des Harzes (Brocken-Granit u. Kahlebergsandstein-Formation), ebenfalls eine signifikante Veränderung, d.h. ein höheres Niveau in der Periode 1981-2000. Die oben diskutierte Anpassung von BFI-Werten und die Übertragung von HÜK200 auf GK50 haben auf die Ergebnisse des U-Tests keinen relevanten Einfluss. Vielmehr werden die zeitlichen Muster in der Grundwasserneubildung hauptsächlich durch die interannuellen Schwankungen des Niederschlags beeinflusst. 


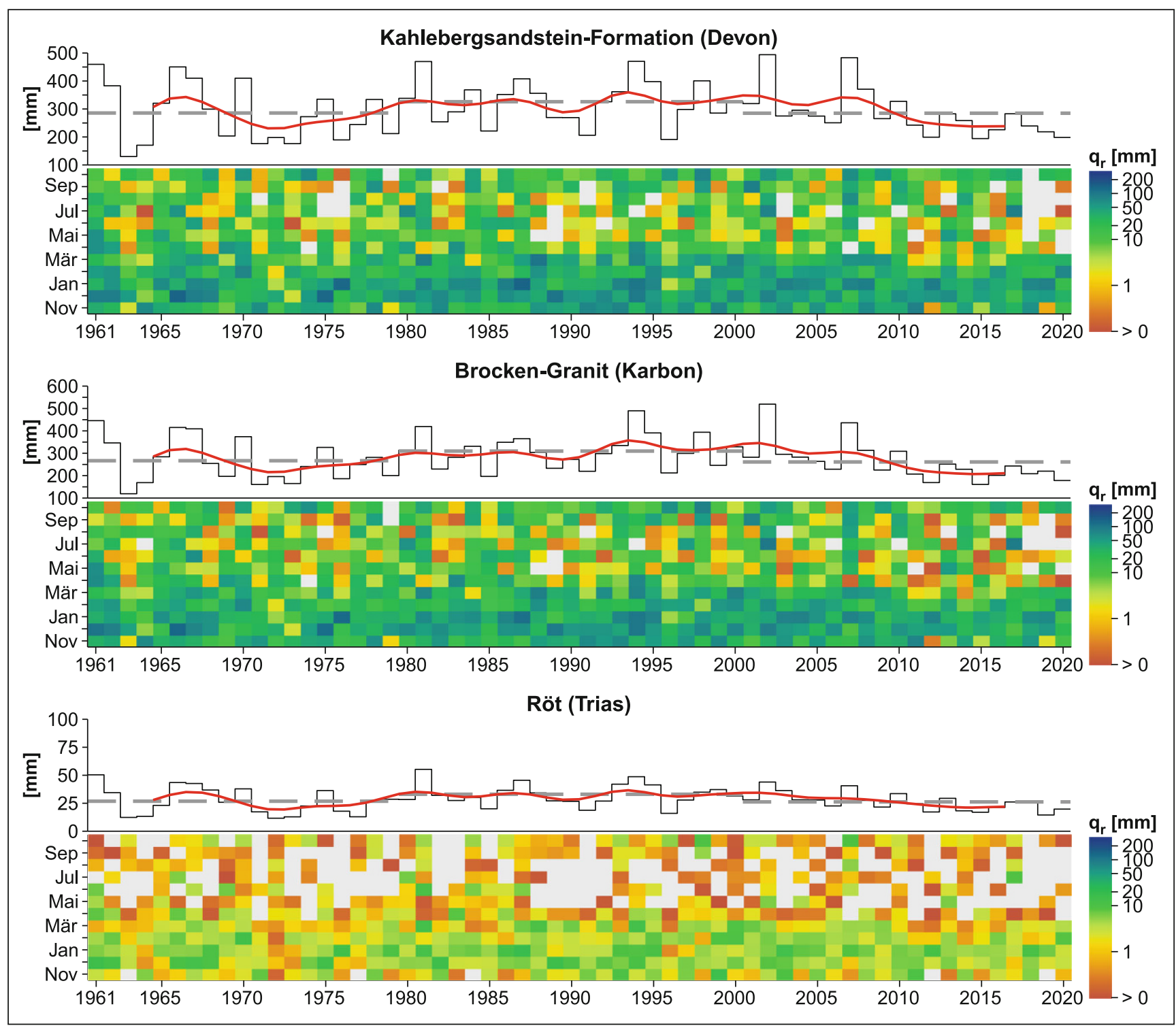

Abb. 5 Zeitreihendarstellung der Grundwasserneubildung in den als Fallbeispielen diskutierten geologischen Einheiten

Fig. 5 Time series of groundwater recharge in the example geological units

\section{Ausblick}

Die am LBEG mit der Modellversion mGROWA18 für Niedersachsen simulierte Grundwasserneubildung ist mittlerweile fester Bestandteil der landesweiten Planungsgrundlagen zur Grundwasserbewirtschaftung. Für diese Modellversion wurde auf Basis der geologischen Information in der GK50 eine räumlich präzisierte Verteilung von BFI-Werten geschaffen, die zum großen Teil aus den in der wasserwirtschaftlichen Praxis schon bewährten BFI-Werten älterer Modellversionen stammen. Die BFI-Werte in mehreren geologischen Einheiten mit lokaler Relevanz beispielsweise für Grundwasserdargebotsbetrachtungen wurden korrigiert. Diese Korrektur war aufgrund zusätzlicher Information in der GK50 sowie aufgrund von Expertenwissen zu den lokalen Grundwasserverhältnissen möglich.

Flächendeckende großräumige Wasserhaushaltsmodelle werden immer wieder mit der Problematik konfrontiert, auch belastbare Ergebnisse für Teilräume eines Modellgebietes zu liefern, in dem bezüglich der Parameter relativ große Unsicherheiten bestehen. Aus wasserwirtschaftlicher Perspektive müssen jedoch auch für solche Fälle Aussagen getroffen werden. Die Ursachen für diese Unsicherheiten sind meist sehr vielschichtig, z.B. Defizite in der räumlichen Auflösung der Datengrundlagen, fehlende Validierungs- und Kalibrierungsmöglichkeiten in Form von $\mathrm{Ab}$ flussganglinien oder auch lokal wenig belastbare Ergebnisse aus empirisch-statistischen Parametrisierungsverfahren. 


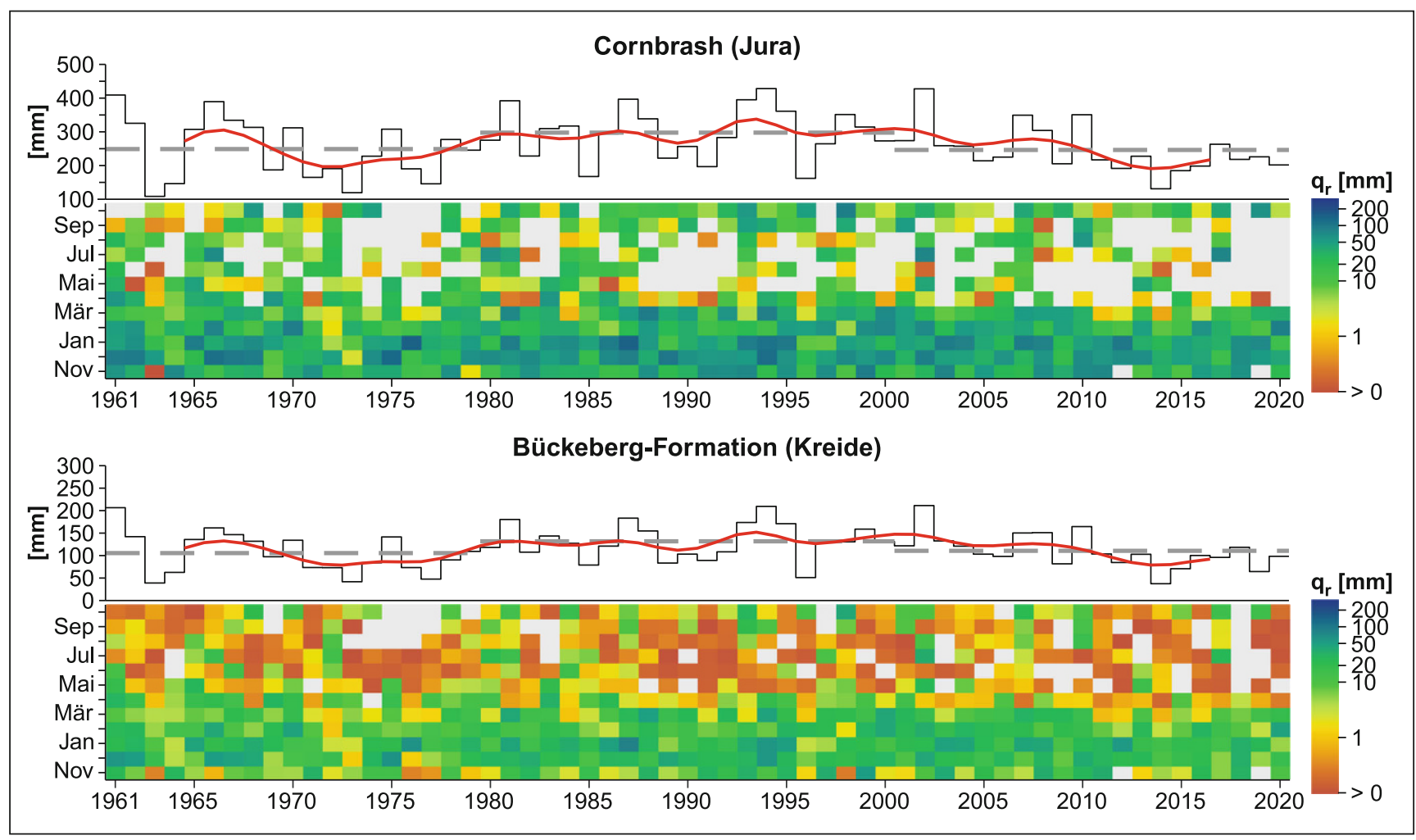

Abb. 5 (Forsetzung) Zeitreihendarstellung der Grundwasserneubildung in den als Fallbeispielen diskutierten geologischen Einheiten

Fig. 5 (continued) Time series of groundwater recharge in the example geological units

Daher sollte die räumliche Verteilung von BFI-Werten in großräumigen Wasserhaushaltsmodellen kontinuierlich auf bestehende Unsicherheiten überprüft und mit zunehmendem Kenntnisstand aus der geologischen Kartierung, durch Erkenntnisse aus Detailstudien beispielsweise in Wasserrechtsverfahren und auch auf Basis von gesichertem Expertenwissen angepasst werden.

Funding Open Access funding enabled and organized by Projekt DEAL.

Open Access Dieser Artikel wird unter der Creative Commons Namensnennung 4.0 International Lizenz veröffentlicht, welche die Nutzung, Vervielfältigung, Bearbeitung, Verbreitung und Wiedergabe in jeglichem Medium und Format erlaubt, sofern Sie den/die ursprünglichen Autor(en) und die Quelle ordnungsgemäß nennen, einen Link zur Creative Commons Lizenz beifügen und angeben, ob Änderungen vorgenommen wurden.

Die in diesem Artikel enthaltenen Bilder und sonstiges Drittmaterial unterliegen ebenfalls der genannten Creative Commons Lizenz, sofern sich aus der Abbildungslegende nichts anderes ergibt. Sofern das betreffende Material nicht unter der genannten Creative Commons Lizenz steht und die betreffende Handlung nicht nach gesetzlichen Vorschriften erlaubt ist, ist für die oben aufgeführten Weiterverwendungen des Materials die Einwilligung des jeweiligen Rechteinhabers einzuholen.

Weitere Details zur Lizenz entnehmen Sie bitte der Lizenzinformation auf http://creativecommons.org/licenses/by/4.0/deed.de.

\section{Literatur}

Ad-hoc-Arbeitsgruppe Hydrogeologie: Regionale Hydrogeologie von Deutschland. Geologisches Jahrbuch, Bd. A 163. Schweizerbart, Stuttgart (2016)

Arnold, J.G., Allen, P.M.: Automated methods for estimating baseflow and ground water recharge from streamflow records. J. Am. Water. Resour. Assoc. 35, 411-424 (1999)

Beven, K.J.: Rainfall-runoff modelling: the primer. John Willey \& Sons, ltd, Chichester (2000)

BGR: Hydrogeologische Übersichtskarte von Deutschland 1:200.000 (2003). unveröff. Entwurf

Bloomfield, J.P., Allen, D.J., Griffiths, K.J.: Examining geological controls on baseflow index (BFI) using regression analysis: an illustration from the Thames Basin, UK. J Hydrol 373, 164-176 (2009)

CONSULAQUA Hildesheim: Geo-Infometric: Hydrogeologisches Gutachten zur Erneuerung der Wasserrechte für die Grundwassergewinnungen Engern, Ahe, Kohlenstädt und Großenwieden der Schaumburger Trinkwasser Verbund- und Aufbereitungsgesellschaft (2015). Dokumentation des numerischen Grundwassermodells (unveröff.)

Demuth, S.: Untersuchungen zum Niedrigwasser in West-Europa. Institut für Hydrologie, Universität Freiburg, Freiburg (1993)

Dörhöfer, G., Josopait, V.: Eine Methode zur flächendifferenzierten Ermittlung der Grundwasserneubildungsrate. Geol. Jb. C27, 45-65 (1980)

Dörhöfer, G., Kunkel, R., Tetzlaff, B., Wendland, F.: Der natürliche Grundwasserhaushalt von Niedersachsen. In: Dörhöfer, G., Röhm, H. (Hrsg.) Arbeitshefte Wasser, Bd. 1, S. 109-167. Schweizerbart'sche, Stuttgart (2001a) 
Dörhöfer, G., Hannappel, S., Voigt, H.-J.: Die Hydrogeologische Übersichtskarte von Deutschland (HÜK 200). Z. Angew. Geol. 47(3+4), 153-159 (2001b)

DWD: Klimareport Niedersachsen (2018). https://www.dwd.de/DE/ leistungen/klimareport_ns/klimareport_ns_download_2018.html (Erstellt: 14. Juni 2018), Zugegriffen: 20. Juni 2018

Eckhardt, K.: A comparison of baseflow indices, which were calculated with seven different baseflow separation methods. J Hydrol 352, 168-173 (2008)

Elbracht, J., Meyer, R., Reutter, E.: Hydrogeologische Räume und Teilräume in Niedersachsen, 2. Aufl. GeoBerichte, Bd. 3. Landesamt für Bergbau, Energie und Geologie, Hannover (2016)

Elbracht, J., Ertl, G., Leßmann, B.: Nachrichtenteil. Grundwasser 23(3), 257-274 (2018)

Engel, N., Müller, U., Schäfer, W.: BOWAB - Ein Mehrschicht-Bodenwasserhaushaltsmodell. In: Müller, U. (Hrsg.) Klimawandel und Bodenwasserhaushalt GeoBerichte, Bd. 20, S. 85-98. Landesamt für Bergbau, Energie und Geologie, Hannover (2012)

Ertl, G., Bug, J., Elbracht, J., Engel, N., Herrmann, F.: Grundwasserneubildung von Niedersachsen und Bremen. Berechnungen mit dem Wasserhaushaltsmodell mGROWA18. GeoBerichte, Bd. 36. Landesamt für Bergbau, Energie und Geologie, Hannover (2019)

Freeze, R.A., Cherry, J.A.: Groundwater. Prentice-Hall, Englewood Cliffs, New Jersey (1979)

Haberlandt, U., Klöcking, B., Krysanova, V., Becker, A.: Regionalisation of the base flow index from dynamically simulated flow components - a case study in the Elbe River Basin. J. Hydrol. Reg. Stud. 248, 35-53 (2001)

Herrmann, A., Schumann, S.: Untersuchung des Abflussbildungsprozesses als Kontrollmechanismus für den Gebietswasserumsatz des Oberharzer Einzugsgebiets Lange Bramke. Hydrol. Wasserbewirtsch. 53(2), 64-79 (2009)

Herrmann, F., Chen, S., Heidt, L., Elbracht, J., Engel, N., Kunkel, R., Müller, U., Röhm, H., Vereecken, H., Wendland, F.: Zeitlich und räumlich hochaufgelöste flächendifferenzierte Simulation des Landschaftswasserhaushalts in Niedersachsen mit dem Model mGROWA. Hydrol Wasserbewirtsch 57, 206-224 (2013)

Heunisch, C., Caspers, G., Elbracht, J., Langer, A., Röhling, H.-G., Schwarz, C., Streif, H.: Erdgeschichte von Niedersachsen: Geologie und Landschaftsentwicklung. GeoBerichte, Bd. 6. Landesamt für Bergbau, Energie und Geologie, Hannover (2007)

Hinze, C., et al.: Geologische Karte von Niedersachsen 1:25 000. Erläuterungen zu Blatt Clausthal-Zellerfeld Nr. 4128. Niedersächsisches Landesamt für Bodenforschung, Hannover (1971)

Jordan, H., Brunotte, E., Büchner, K.-H., Dörhöfer, G., Garleff, K., Irrlitz, W., Kosmahl, W., Lüders, R., Rost, A.: Geologische Karte von Niedersachsen 1:25 000. Erläuterungen zu Blatt Nr. 4325 Nörten-Hardenberg. Niedersächsisches Landesamt für Bodenforschung, Hannover (1984)

Kottek, M., Grieser, J., Beck, C., Rudolf, B., Rubel, F.: World map of the Köppen-Geiger climate classification updated. Meteorol. Zeitschr. 15(3), 259-263 (2006)

Kunkel, R., Wendland, F.: Der Landschaftswasserhaushalt im Flußeinzugsgebiet der Elbe - Verfahren, Datengrundlagen und Bilanzgrößen (1998). http://hdl.handle.net/2128/12189. Zugegriffen: 20. Juni 2021

Kunkel, R., Bogena, H., Tetzlaff, B., Wendland, F.: Digitale Grundwasserneubildungskarte von Niedersachsen, Nordrhein-Westfa- len, Hamburg und Bremen: Erstellung und Auswertungsbeispiele. Hydrol. Wasserbewirtsch. 50(5), 212-219 (2006)

LBEG: Geologische Karte von Niedersachsen 1:50.000 - Grundkarte (GK 50) (2007). http://nibis.lbeg.de/cardomap3/public/ogc. ash $x$ ?NodeId $=146 \&$ Service $=$ WMS $\&$ Request $=$ GetCapabilities $\&$, Zugegriffen: 20. Juni 2021

LBEG: Hydrogeologische Karte von Niedersachsen 1:50 000 - Mittlere jährliche Grundwasserneubildungsrate 1981-2010, Methode mGROWA18 (2019). https://nibis.lbeg.de/cardomap3/public/ogc. ash $x$ ?NodeId $=1107 \&$ Service $=$ WMS $\&$ Request $=$ GetCapabilities $\&$, Zugegriffen: 20. Juni 2021

Lemke, D., Elbracht, J.: Grundwasserneubildung in Niedersachsen. Ein Vergleich der Methoden Dörhöfer \& Josopait und GROWA06V2. GeoBerichte, Bd. 10. Landesamt für Bergbau, Energie und Geolo, Hannover (2008)

Mann, H.B., Whitney, D.R.: On a test of whether one of two random variables is stochastically larger than the other. Ann. Math. Stat. 18, 50-60 (1947)

Mauget, S.A.: Multidecadal regime shifts in U.S. streamflow, precipitation, and temperature at the end of the twentieth century. J. Climate 16, 3905-3916 (2003)

Naumann, E.: Erläuterungen zur geologischen Karte von Preußen und benachbarten deutschen Ländern 1:25000: Lfg. 265 - Blatt Eldagsen Nr. 2088. Geologische Landesanstalt, Berlin (1927)

Partington, D., Brunner, P., Simmons, C.T., Werner, A.D., Therrien, R., Maier, H.R., Dandy, G.C.: Evaluation of outputs from automated baseflow separation methods against simulated baseflow from a physically based, surface water-groundwater flow model. J. Hydrol. Reg. Stud. 458-459, 28-39 (2012)

Reutter, E.: Hydrostratigrafische Gliederung Niedersachsens, 2. Aufl. Geofakten, Bd. 21. Landesamt für Bergbau, Energie und Geologie, Hannover (2011)

Scheihing, K.W.: Klimawandel in Niedersachsen und mögliche Folgen für die Grundwasserbewirtschaftung: ein Review. Hydrol. Wasserbewirtsch. 63, 85-97 (2019)

Tallaksen, L.M.: A review of baseflow recession analysis. J. Hydrol. Reg. Stud. 165, 349-370 (1995)

Wendland, F., Kunkel, R., Tetzlaff, B., Dörhöfer, G.: GISbased determination of the mean long-term groundwater recharge in Lower Saxony. Environ Geol 45(2), 273-278 (2003)

Wilcoxon, F.: Individual comparisons by ranking methods. Biomed. Bull. 1, 80-83 (1945)

Wittenberg, H.: Baseflow recession and recharge as nonlinear storage processes. Hydrol. Process. 13, 715-726 (1999)

Wittenberg, H., Aksoy, H., Miegel, K.: Der schnelle Anstieg des Grundwassers nach Starkregen. Hydrol. Wasserbewirtsch. 64(2), 66-74 (2020)

Wundt, W.: Die Kleinstwasserführung der Flüsse als Maß für die verfügbaren Grundwassermengen. In: Grahmann, R. (Hrsg.) Die Grundwässer in der Bundesrepublik Deutschland und ihre Nutzung Forsch. dt. Landeskunde, Bd. 104, S. 47-54. BA für Landeskunde, Remagen (1958)

Hinweis des Verlags Der Verlag bleibt in Hinblick auf geografische Zuordnungen und Gebietsbezeichnungen in veröffentlichten Karten und Institutsadressen neutral. 